\title{
Investigating the Influence of Culture on Proxemic Behaviors for Humanoid Robots
}

\author{
Ghadeer Eresha $^{1}$, Markus Häring ${ }^{2}$, Birgit Endrass ${ }^{2}$, Elisabeth André ${ }^{2}$ and Mohammad Obaid ${ }^{2,3}$ \\ ${ }^{1}$ Media Engineering and Technology Dept., The German University in Cairo, Egypt \\ ${ }^{2}$ Human Centered Multimedia Lab (HCM), Augsburg University, Germany \\ ${ }^{3}$ HITLab New Zealand, University of Canterbury, New Zealand
}

\begin{abstract}
In social robotics, the behavior of humanoid robots is intended to be designed in a way that they behave in a human-like manner and serve as natural interaction partners for human users. Several aspects of human behavior such as speech, gestures, eye-gaze as well as the personal and social background of the user need therefore to be considered. In this paper, we investigate interpersonal distance as a behavioral aspect that varies with the cultural background of the user. We present two studies that explore whether users of different cultures (Arabs and Germans) expect robots to behave similar to their own cultural background. The results of the first study reveal that Arabs and Germans have different expectations on the interpersonal distance between themselves and robots in a static setting. In the second study, we use the results of the first study to investigate the users' reactions on robots using the observed interpersonal distances themselves. Although the data of this dynamic setting is not conclusive, it suggests that users prefer robots that show behavior that has been observed for their own cultural background before.
\end{abstract}

\section{INTRODUCTION}

Cultural background does not only influence our own behavior but also how the behavior of others is perceived. This is especially crucial for aspects of behavior that are managed at a rather subconscious level of awareness, as they might stay unnoticed at first but leave a negative impression of the interlocutor afterwards. This is illustrated vividly by Pease [1] who describes several social situations where cultural background might lead to misunderstandings.

In a similar manner, computer-based systems might be misunderstood, especially when aiming at simulating human behavior or providing natural interaction. In the research area of intelligent virtual agents, behavioral aspects have widely been researched in relation to cultural background, e.g. [2] [3]. Perception studies showing virtual characters reveal that users seem to prefer agent behavior that reflects their own cultural background for aspects of verbal as well as nonverbal behavior [4][5].

In the field of human-robot interaction, researchers aim on producing humanoid robots that are socially acceptable when interacting with humans. Thus, it seems only natural to include social background such as culture for their behavioral models as well.

In this paper, we investigate interpersonal distance as a behavioral aspect that depends on cultural background. In the following section, we present related work from the field of social robotics. Subsequently, we introduce culture and how it relates to interpersonal distance behavior. Then, we describe two studies: In the first study, we identify expected interpersonal distances in multi-party conversations with robots and humans for different cultures. Later, we transfer the findings from this study into a dynamic application where we investigate the users' reactions to humanoid robots showing the behaviors observed in the first study. Finally, we conclude our work and outline some future work.

\section{RELATED WORK}

Investigating cultural background for humanoid robots is rather unexplored in the domain of social robotics. Exceptions include studies by Bartneck et al. [6][7] and Nomura et al. [8], who have shown that the attitude towards robots depends on cultural background. Despite the fact that these general attitudes are often negative, little has been done to investigate how human-robot interaction can be improved by considering the cultural background of an interaction partner. One of these few studies on cultural adaptive robots is the work done by Rau et al. [9] who investigated how the communication style (i.e. implicit or explicit) and the language (i.e. native language versus English) would affect the acceptance of recommendations made by a robot among Chinese and German users. Their results reveal that Chinese participants evaluated the robot as more likable, trustworthy, and credible when the robot used implicit communication style, while German participants would less likely accept its recommendations.

This example illustrates that small adoptions to cultural preferences already have a positive effect on the whole interaction with a robot. We consider this as a motivation to investigate further aspects of culture-specific behavior.

Proxemic behavior is known to have a strong effect on the perception of an interaction. In 1966, Hall introduced social zones for human social interaction [10]. These zones describe borders of comfortable interpersonal interaction, depending on various factors such as relationship (e.g. friends vs. strangers) and purpose of the interaction (e.g. private conversation vs. public speech). Work done by Walters et al. [11] confirms that humans mostly conform to Hall's social zones when interacting with a robot. However, Walters et al. did not investigate differences in cultural background.

In regard to the question "What is socially acceptable proxemic behavior for robots interacting with humans?", 
Torta et al. [12] presented a behavior-based navigational architecture for the humanoid robot Nao. The idea of this architecture was to safely navigate a robot in a dynamically changing domestic environment, while respecting the user's personal space. Their results suggests that humans have a distinct impression of appropriate proxemic behaviors for robots, however there is no comparison whether this behavior conforms to common rules of human-human interaction.

Mumm and Mutlu [13] explored how people physically and psychologically distance themselves from robots considering four prominent models that offer competing predictions on how individuals respond to attempts by others to change distancing. In an experiment with 60 participants, they manipulated the likability and gaze behavior of a robot, to investigate how these factors affect the participants' physical distance to the robot. They found that participants who disliked the robot compensated for increased gaze by distancing themselves further from it; while those who liked the robot were not affected by increased gaze. Also, participants who liked the robot disclosed more with the robot than those who disliked the robot. Their results allowed Mumm and Mutlu to form a comprehensive theoretical model of humanrobot proxemics to which future designs of human-robot interactions could resort to. Still this model is only applicable to interaction between one human and one robot, while we are interested in proxemic behaviors for robots that are involved in a multi-party conversation.

Takayama and Pantofaru [14] conducted a study in which a human-like size robot approached the participants until they told it to stop, quite similar to the study of Torta et al [12]. In their work, not only the size of the robots was different, but they also added the condition where the participants approached the robot up to a comfortable distance. Furthermore, they were not only interested in the distances themselves, but if factors like pet ownership, experience with robots, and the direction of the robots gaze would influence those distances. Their findings confirm that all of these factors can influence the distances preferred in a humanrobot interaction. On the other hand, they observed that there is no difference in distances whether the robot approaches the human or vise-versa.

The studies mentioned above emphasize that common rules of proxemic behaviors apply to robots, and different factors have to be considered to guarantee comfortable interaction with humans. However, thus far, none of the studies considered the cultural background as a variable for interpersonal distance behaviors and findings are limited to one-one interactions. In this paper we investigates the effects of culture on proxemic behaviors in human-robot conversations.

\section{Culture And Proxemic Behavior}

A very well known dimensional model of culture is introduced by Hofstede et al. [15], whose theory categorizes national cultures into a six dimensional model. Each dimension contains two extreme sides, for which stereotypical behavioral norms are defined. The Individualism dimension is of special interest for our purpose since it is strongly related to interpersonal distance behavior. In this paper, we exemplify our approach for the Arab and German cultures due to availability of participants. These two cultures seem very well suited since behavioral differences in proxemics are expected to be huge. Due to the scores on Hofstede's Individualism dimension, Germany is considered to be an individualistic culture, while the Arab world is considered being collectivistic [15]. In [16], Hofstede et al. investigate cultural dimensions in isolation and describe prototypical behavior for cultures that find themselves on one of the extreme sides. Individualistic cultures, are described to stand free in groups, while collectivistic cultures tend to be physically close, especially to in-groups. This suggests that interpersonal distance should be higher in prototypical German conversations compared to prototypical Arab ones.

This idea is supported by Hall's cultural dichotomy that categorizes cultural groups into so-called high- and lowcontact cultures [10], that largely differ in behavioral aspects such as interpersonal distance, eye-gaze or body contact. Germany belongs to the medium-contact group while Arabia belongs to the high-contact group. Regarding proxemics behavior, in high-contact cultures close interactions are common, while in low-contact cultures wider interpersonal space is appropriate.

For human-human interaction, studies have been conducted to explore appropriate interpersonal distances in conversational settings across cultures. According to TingToomey [17], the average conversational distance for European Americans is approximately 20 inches $(\approx 51 \mathrm{~cm})$, while the ideal conversational distance for two Arabs is only approximately $9-10$ inches $(\approx 23-25 \mathrm{~cm})$. Although these numbers can serve as a guideline to implement behavioral models for humanoid robots, it is unlikely that they can be transfered directly, as common humanoid robots are much smaller than the average human body size. Therefore, in the following section, we introduce a study that seeks to find the expected interpersonal distances in robot-robot as well as robot-human settings for the two targeted cultures.

\section{EXPERIMENTAL EVALUATION}

In this section, we describe a controlled experiment that investigates the human-robot interpersonal distances in conversational scenarios. We recruit participants from an Arab and a German cultural background to investigate the influence of culture on the human-robot proxemic behaviors. In line with previous literature on human-human interaction behaviors (described in Section III), we expect that the interpersonal distance to be higher in prototypical German settings compared to prototypical Arab ones.

\section{A. Experimental Design}

1) Scenarios: In our experiment, we seek to find interpersonal distances that are perceived as being appropriate in the German and Arab cultures, first in dyadic conversations between robots only and second in triadic conversations 
between two robots and a participant. Therefore, participants of the target cultural backgrounds had to perform two tasks:

Robot-Robot scenario $(R R)$ : In this scenario, each participant was asked to imagine two robots talking to each other in a friendly conversation. One robot was already positioned in the experimental room. As a task, the participant was asked to place the second robot in a comfortable position and distance according to his or her personal preference (see Figure 1 for an example positioning). For this scenario, we hypothesize that participants with a German cultural background arrange robots at a larger distance to each other compared to participants with an Arab cultural background (Hypothesis 1).

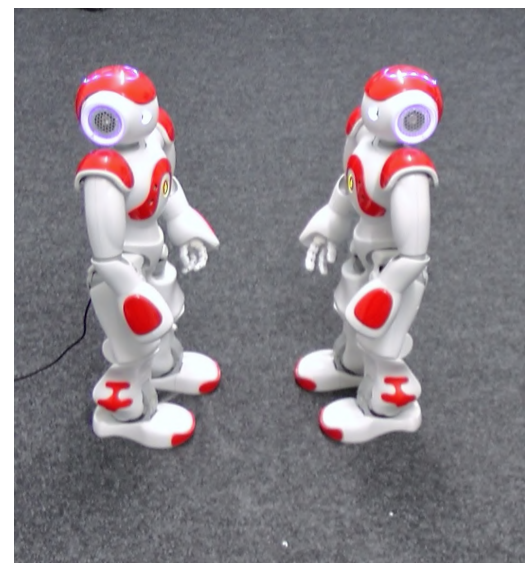

Fig. 1. Example of a Robot-Robot conversation

Human-Robot-Robot scenario (HRR): In this scenario, participants were part of the imaginary conversation with the two robots. As in the previous scenario, the first robot was positioned in the room already. Each participant was asked to place the second robot at a comfortable position and distance according to their own preference. As a next step, the participants were asked to comfortably place themselves to be part of this conversation (see Figure 2). For the scenario, we hypothesize that participants with an Arab cultural background will arrange robots and themselves at a closer interpersonal distance compared to participants with a German cultural background (Hypothesis 2).

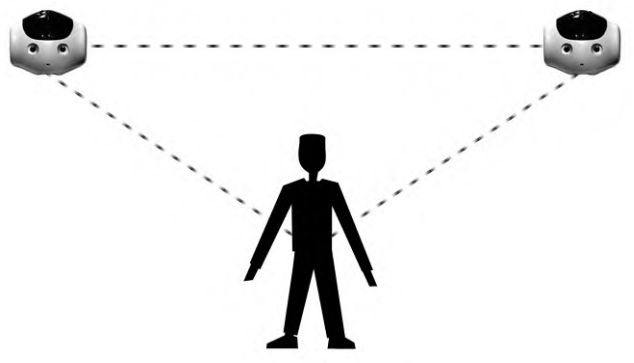

Fig. 2. Example of a Human-Robot-Robot conversation
2) Robots: In the experiment, we used the Nao robot from Aldebaran Robotics ${ }^{1}$. Its humanoid appearance features two feet, two arms and a head, all linked to the trunk. Although some aspects of the head resemble eyes, ears and a mouth, the robot has no facial expressions. The robot has a height of 56 centimeters. The robots were switched on during the study and the LEDs of the robots indicated that they were running, though, they did not speak or move during the experiment.

3) Procedure: The experiment was conducted in a closed room with controlled lighting and no outside distraction. Only the experimenter, two robots, and one participant were present in the room. Participants were lead into the experiment room where the experimenter told them that they were going to accomplish two tasks with robots. Then they were given a detailed written description of the first task. Upon indicating that they understood the instructions, they were given the second robot to place it in relation to the first. After completing the first task, the participants were asked to leave the room and measurements were recorded (Section IV-C). Later, they entered the experiment room again and were asked to perform the second task. The duration of the session was approximately 10 minutes and the order of the two tasks were randomized.

\section{B. Participation}

A total of 12 German participants (6 males and 6 females) and 12 Arab participants ( 6 males and 6 females) took part in the experiment. As body size could matter in this study, we asked participants for their height. The average height of the cultural groups were similar, with an average size of 170.25 $\mathrm{cm}$ for the Arab group $(179.50 \mathrm{~cm}$ for males and $161.00 \mathrm{~cm}$ for females) and $171.17 \mathrm{~cm}$ for the German group (179.50 $\mathrm{cm}$ for males and $162.83 \mathrm{~cm}$ for females).

\section{Measurements}

After every trial of the RR task, the distance between the two robots was measured. After every trial of the HRR task, the distance between the two robots and the distance between the participant and both robots were measured.

\section{Results and Discussion}

The data analysis started with manipulation checks for the measurements of the Arab and German participants using mixed factorial ANOVA and planned contrasts, with the different types of distances (Human to Robot (HR) and Robot to Robot (RR)) in the different interaction scenarios (RobotRobot and Human-Robot-Robot) as Within-Subjects factor and the cultural background as the Between-Subjects factor.

For the type of distance the test revealed a highly significant $(p<.001)$ main effect, $F(2,44)=39.31$, and a significant $(p<.05)$ interaction effect between the type of distance and the cultural background of the participant, $F(2,44)=4.07$. This means that independent from the cultural background the distances in the two interaction scenarios vary significantly, but the cultural background has a significant effect on the variation of distances. In Table I,

\footnotetext{
${ }^{1}$ http://www.aldebaran-robotics.com/en/
} 
you can see the exact distances in centimeters for every type of distance and for every cultural group.

As you can see the measurements in the RR scenario do not support our first hypothesis. Both the Arab and German participants placed the robots at a average distance of about $40 \mathrm{~cm}(p>.05)$.

In the HRR scenario, Arabs and Germans placed the two robots (RR) at approximately the same distance from each other. Although the distances are not significantly different between the cultural groups, the difference in distance between the two scenarios $(40 \mathrm{~cm}$ to $60 \mathrm{~cm})$ is significant $(p<.001)$. So both cultures expect to the same extent that the interpersonal distance increases when a human is involved for a multi-party conversation.

\begin{tabular}{|c|c|c|c|c|}
\multicolumn{1}{c|}{} & \multicolumn{2}{c|}{ Arab } & \multicolumn{2}{c|}{ German } \\
\cline { 2 - 5 } \multicolumn{1}{c|}{} & Mean & SD & Mean & SD \\
\hline Robot-Robot & 39.75 & 8.34 & 42.33 & 8.19 \\
\hline HRR - RR & 60.17 & 18.59 & 59.08 & 17.68 \\
\hline HRR - HR & 65.79 & 19.69 & 85.63 & 26.30 \\
\hline
\end{tabular}

TABLE I

RESULTS OF THE STUDY (IN CENTIMETER, HRR:

HUMAN-ROBOT-Robot SCENARIO, HR: DISTANCE OF HUMAN TO ROBOT, RR: DISTANCE OF ROBOT TO ROBOT)

With regard to our second hypothesis, our analysis of the the HRR scenario confirmed that Arabs arrange themselves at a significantly closer distance to the robots than the German participants $(p<.01)$.

From this data we conclude two different conversational settings for Arabs and Germans depicted in Figure 3.

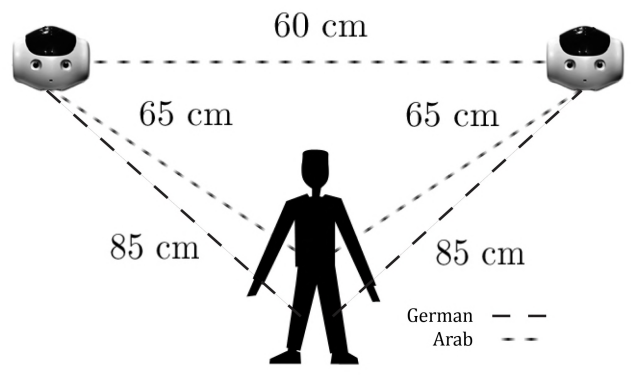

Fig. 3. Distance of Arab and German participants to the robots in a humanrobot-robot conversation

Interestingly, in the Arab setting the interpersonal distances from human to robots (HR) and robot to robot (RR) are almost equal, but they are significantly different in the German setting. One might conclude that Arabs and Germans arrange themselves to the robots (HR) according to their cultural background, but the interpersonal distance between the robots (RR) seems not to follow the same rules. These observations might indicate that both the German and the Arab participants considered the robots as not being a member of their own culture, German or Arab.
In summary, we can say that our findings are consistent with the literature about human interaction for the HRR scenario. Our results support the assumption that Arabs and Germans expect interpersonal distances between humans and robots similar to multi-party human-human interactions. Interestingly, our results do not support the assumption that users will assign their own behavior to the robots, as there were no differences in the interpersonal distances in the RR scenario.

\section{PRELIMINARY STUDY - FROM A STATIC TO A DYNAMIC SCENARIO}

The first study revealed specific conversational settings for multi-party human-robot conversations with Arab and German users, and for robot-robot conversations. So far, participants arranged the setting according to their own preferences and expectations, and were not exposed to a setting yet in which the robots actively showed proxemic behavior. To investigate whether users prefer robots which arrange themselves in respect to the users' cultural background, we designed a study that integrates both of our initial scenarios (RR and HRR interaction) into a dynamic setting, in which the robots actually move towards the user.

Therefore, we implemented a scenario in which the two robots are initially engaged in a one-one discussion among themselves (which correlates to the first interaction scenario in section IV-A.1), then the robots notice the user and approach him or her for a conversation.

\section{A. Experimental Design}

For the dynamic scenario, we use the distances collected in the first study. In the experiment, the robots start their initial conversation with an interpersonal distance of $40 \mathrm{~cm}$ (RR). The static study revealed a significant increase of this distance in the HRR setting, so this distance changes to 60 $\mathrm{cm}$ while the robots approach the participant. We arranged two settings for the approaching of the robots: in the Arab setting, the robots stop in front of the participant when reaching an interpersonal distance of $65 \mathrm{~cm}$, in the German setting when reaching an interpersonal distance of $85 \mathrm{~cm}$. Figure 4 schematically describes the German setting.

To simulate realistic interaction with the robots we added speech and minimal gaze behavior. In other perception studies, gibberish is used to eliminate the chance that participants are influenced by the content of the dialog [18]. For the same reason, gibberish was produced using the Text-To-Speech module of Nao.

The robots talk to each other at the start of the scenario, then they approach the participant, greet by saying "hello" and continue to speak in gibberish. The greeting was the only utterance the participants were suppose to understand, so they get the impression that the robots are talking to them and do not simply continue talking to each other.

The simple gaze behavior was added to give participants the impression that the robots are attentive. The robots looked at the user before approaching them, to signalize that they became aware of the presence of the user and intentionally 


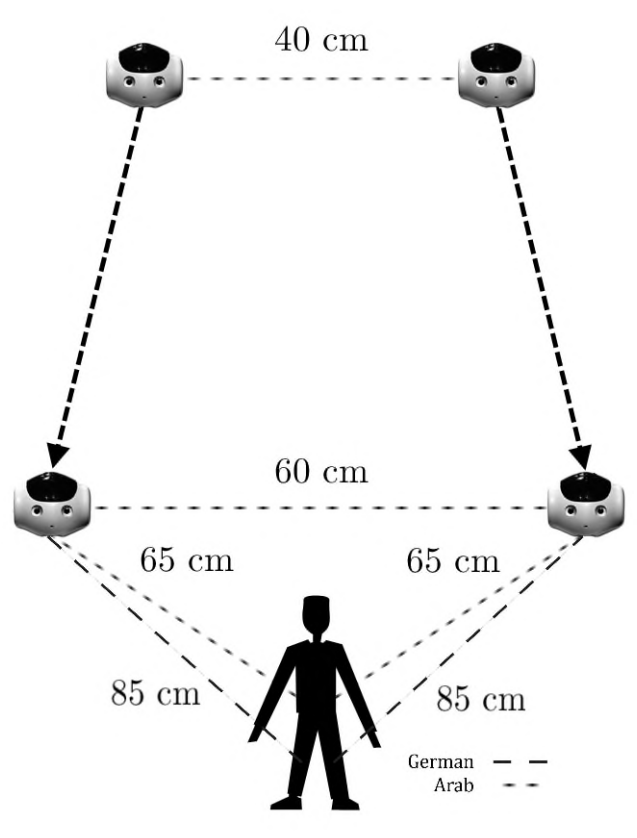

Fig. 4. Schemata with distances in the German setting compared to the Arab setting of the Human-Robot-Robot conversation

decided to start approaching. If possible, the robots keep an eye contact with the user while approaching, and during the short dialog with the user after they have reached the interpersonal distance of the current setting. Please note that the face detection which is necessary for the gaze behavior, can be negatively affected by different individual factors such as glasses, head scarfs or the vibrations caused while the robot is walking.

While the dialog is scripted for each robot individually, the proxemic and gaze behavior is similar for both. However, the robots act (look at and approach the user) with a small delay between each other because a fully synchronous behavior would appear mechanical and unnatural.

\section{B. Participation}

To evaluate whether the cultural groups prefer robot behavior that reflects their own cultural background, the participants experienced both proxemic settings (German and Arab). In the experiment, 12 Arab and 12 German users participated (6 male and 6 female in each group), while they were mostly the same participants as in the static study. Keeping in mind that different cultures can have different attitudes towards robots which could effect the proxemic behavior, we measured the participants level of anxiety towards robots before the actual interaction, using the Negative Attitude toward Robots Scale (NARS)[19].

The robots started their conversation 1.5 meters away from the point where the participant was located in the beginning of the experiment. The robots noticed the user after a fixed time duration and started to approach him or her. We counterbalanced the order of the settings, and after each setting the users were asked to rate on a 5-Point Likert-scale several subjective items such as "The robots seemed friendly to me", "The robots seemed interested in me", "The robots seemed cold / reserved to me", "The robots seemed obtrusive / pushy to me", "The robots seemed to like me", and "I liked the robots". Additionally, we asked them what they think the robots were talking about, to get a deeper insight into how the participants experienced the situation. Finally, we asked them what in their opinion the difference between the two settings was, which one they preferred and why.

\section{Results and Discussion}

The most interesting question in this setting was: which of the scenarios was preferred more by each of the cultural groups. In both groups, half of the participants preferred the Arab setting and the other half the German setting, which did not match our expectations. A look at the comments and answers of the questionnaires revealed that half of the participants were not aware of the difference in distance and some of them stated that they felt quite uncomfortable during the experiment. Further investigations in this matter revealed that the gibberish speech and occasional errors in gaze behavior unsettled several users. Two German participants stated that they thought the robots were planning to attack them. Unfortunately, in a few cases the face detection had positive detections in the environment which caused the robots not to look at the user anymore. Some participants perceived this behavior as rude. There were also users who mentioned that the robots were acting too synchronized, despite the delay we implemented for the robots behaviors.

After learning about these drawbacks, we focused on participants who stated that they noticed a difference in interpersonal distance and where not distracted by other factors. A closer look at the data of this subgroup revealed a tendency towards the cultural setting that was designed to resemble their own cultural background. Five out of six Arab participants who were aware of the difference, preferred the Arab setting, while four out of seven Germans who were aware of the difference preferred the German setting. Another German participant said that he did not prefer any of the settings, so for the German participants there is still a ratio of $4: 2$ in favor for the German setting compared to the Arab setting.

Of course with a population of that size and the reported drawbacks of the study, the data of our second study does not confirm our assumption. However, the fact that there is a tendency into the right direction among those participants who were aware of the behavioral difference, gives some interesting insights. We learned few lessons, such that we have to consider other components and factors of the interaction more carefully, so that the user is not distracted too much or placed in a too uncomfortable situation. We were surprised that some users were afraid of the robots, since the initial reaction to the robots was usually quite positive in our former studies because of their rather cute appearance. The qualitative data did not reveal significant differences in the subjective perception of the two scenarios. 
In regard to other relevant factors, there is evidence that the level of anxiety influences the comfortable distance in human-robot interaction [20]. Our initial analysis of the NARS values revealed no significant difference in the levels of anxiety for our cultural groups $\left(N A R S_{A r a b}=3.19\right.$, $\left.N A R S_{\text {German }}=3.25, p>.05\right)$. With regard to our challenging results we conducted a factorial ANOVA to take a closer look at the NARS values for those who preferred the Arab setting (a closer interaction) in contrast to those who preferred the German setting (an interaction further apart). In line with the literature the test revealed that those who chose the Arab setting had a significant $(p<0.05)$ lower level of initial anxiety towards robots $\left(N A R S_{\text {close }}=2.75\right)$ than those who later preferred the German setting $\left(N A R S_{f a r}=\right.$ 3.68). This trend is independent of the cultural background and suggest that regardless of cultural conventions personal factors have a very strong effect on the proxemic behavior.

\section{CONCLUSion AND Future Work}

In this paper, we presented two studies investigating the influence of culture on proxemic behavior for social humanoid robots. The goal of the first study was to identify the expected interpersonal distances in Robot-Robot and Human-Robot-Robot interactions with Arab and German participants. The data collected is consistent with literature on human-human interaction, in particular where the Arab participants preferred a closer interpersonal distance in multiparty conversations than Germans. In addition, we conducted a second study by developing a two conversational settings, one containing a prototypical Arab distance behavior and another containing a prototypical German distance behavior.

In the second study, we transferred these settings to a dynamic application, in which the robots approached the user with different proxemic behaviors. The results showed that unexpected gaze behavior or speech can place the user in an uncomfortable situation, which can distract him or her from the actual study. Still we found a tendency among those participants who were aware of the difference in distance behavior to prefer proxemic behavior that reflects their own cultural background.

Considering our experiences, we plan to collect data with a similar design to the study of Torta et al. [12] in which the robot approached the users until they told it to stop to assure that participants do not feel uncomfortable. We plan to conduct the study both ways round: with the user approaching the robots, and the robots approaching the user; with a focus on cultural differences. Based on the outcome we will be able to refine our implementations of the proxemic behaviors for humanoid robots.

\section{ACKNOWLEDGMENT}

This research was funded by EC under grant agreement eCUTE (FP7-ICT-257666). The authors are solely responsible for the content of this publication. It does not represent the opinion of the EC, and the EC is not responsible for any use that might be made of data appearing therein.

\section{REFERENCES}

[1] A. Pease, Body Language: How to read other's thoughts by their gestures. London: Sheldon Press, 1993.

[2] W.-L. Johnson and A. Valente, "Tactical Language and Culture Training Systems: Using Artificial Intelligence to Teach Foreign Languages and Cultures," in Innovative Applications of Artificial Intelligence (IAAI 2008). Association for the Advancement of Artificial Intelligence (AAAI), 2008, pp. 1632-1639.

[3] R. Aylett, A. Paiva, N. Vannini, S. Enz, E. André, and L. Hall, "But that was in another country: agents and intercultural empathy," in Proc. of 8th Int. Conf. on Autonomous Agents and Multiagent Systems (AAMAS 2009), C. Sierra, C. Castelfranchi, K. S. Decker, and J. S. Sichman, Eds., Budapest, Hungary, 2009.

[4] B. Endrass, E. André, M. Rehm, and Y. Nakano, "Investigating culturerelated aspects of behavior for virtual characters," Autonomous Agents and Multi-Agent Systems, 2013.

[5] M. Obaid, I. Damian, F. Kistler, B. Endrass, J. Wagner, and E. André, "Cultural behaviors of virtual agents in an augmented reality environment," in Proceedings of the 12th international conference on Intelligent Virtual Agents, ser. IVA'12. Berlin, Heidelberg: SpringerVerlag, 2012, pp. 412-418.

[6] C. Bartneck, T. Nomura, T. Kanda, T. Suzuki, and K. Kato, "Cultural differences in attitudes towards robots," Proceedings of the Symposium on Robot Companions: Hard Problems and Open Challenges in RobotHuman Interaction, 2005.

[7] C. Bartneck, T. Suzuki, T. Kanda, and T. Nomura, "The influence of people's culture and prior experiences with aibo on their attitude towards robots," AI \& SOCIETY, vol. 21, pp. 217-230, 2007.

[8] T. Nomura, T. Suzuki, T. Kanda, J. Han, N. Shin, J. Burke, and K. Kato, "What people assume about humanoid and animal-type robots: cross-cultural analysis between japan, korea, and the united states," International Journal of Humanoid Robotics, vol. 05, no. 01, pp. 25-46, 2008.

[9] P. P. Rau, Y. Li, and D. Li, "Effects of communication style and culture on ability to accept recommendations from robots," Comput. Hum. Behav., vol. 25, no. 2, pp. 587-595, Mar. 2009.

[10] E. T. Hall, The Hidden Dimension. Doubleday, 1966.

[11] M. Walters, K. Dautenhahn, R. te Boekhorst, K. L. Koay, C. Kaouri, S. Woods, C. Nehaniv, D. Lee, and I. Werry, "The influence of subjects' personality traits on personal spatial zones in a human-robot interaction experiment," in Robot and Human Interactive Communication, 2005. ROMAN 2005. IEEE International Workshop on, aug. 2005 , pp. $347-352$.

[12] E. Torta, R. H. Cuijpers, J. F. Juola, and D. van der Pol, "Design of robust robotic proxemic behaviour," in Proceedings of the Third international conference on Social Robotics, ser. ICSR'11. Berlin, Heidelberg: Springer-Verlag, 2011, pp. 21-30.

[13] J. Mumm and B. Mutlu, "Human-robot proxemics: physical and psychological distancing in human-robot interaction," in Proceedings of the 6th international conference on Human-robot interaction, ser. HRI '11. New York, NY, USA: ACM, 2011, pp. 331-338.

[14] L. Takayama and C. Pantofaru, "Influences on proxemic behaviors in human-robot interaction," in Intelligent Robots and Systems, 2009. IROS 2009. IEEE/RSJ International Conference on, 2009, pp. 54955502.

[15] G. Hofstede, G.-J. Hofstede, and M. Minkov, Cultures and Organisations. SOFTWARE OF THE MIND. Intercultural Cooperation and its Importance for Survival. McGraw Hill, 2010.

[16] G. J. Hofstede, P. B. Pedersen, and G. Hofstede, Exploring Culture Exercises, Stories and Synthetic Cultures. Yarmouth, United States: Intercultural Press, 2002.

[17] S. Ting-Toomey, Communicating across cultures. New York: The Guilford Press, 1999.

[18] B. Endrass, M. Rehm, A.-A. Lipi, Y. Nakano, and E. André, "Culturerelated Differences in Aspects of Behavior for Virtual Characters across Germany and Japan," in Proc. of 10th Int. Conf. on Autonomous Agents and Multiagent Systems (AAMAS 2011), L. Sonenberg, P. Stone, K. Tumer, and P. Yolum, Eds., 2011, pp. 441-448.

[19] T. Nomura, T. Kanda, and T. Suzuki, "Experimental investigation into influence of negative attitudes toward robots on humanrobot interaction," AI \& SOCIETY, vol. 20, no. 2, pp. 138-150, 2006.

[20] T. Nomura, T. Shintani, K. Fujii, and K. Hokabe, "Experimental investigation of relationships between anxiety, negative attitudes, and allowable distance of robots," in Proc. of the 2nd IASTED Intl. Conf. on Human Computer Interaction. ACTA Press, 2007, pp. 13-18. 